\title{
Codependence, Contradependence, Gender-Stereotyped Traits, Personality Dimensions and Problem Drinking
}

\author{
Catherine A. Hawkins ${ }^{1, *}$, Raymond C. Hawkins II $^{2}$ \\ ${ }^{1}$ School of Social Work, Texas State University, San Marcos, TX, 78666,USA \\ ${ }^{2}$ Fielding Graduate University \& Psychology Dept., University of Texas at Austin, Austin, TX, 78712, USA \\ *Corresponding Author: ch11@txstate.edu
}

Copyright (C) 2014 Horizon Research Publishing All rights reserved.

\begin{abstract}
This study explored the relationship between codependence assessment scales, gender, positive and negative gender-stereotyped traits, and other measures of personality and problem drinking. These instruments were administered to a sample of 208 American undergraduates. The results revealed no gender differences on the codependence measures. Students reporting a positive family history of alcohol problems scored significantly higher on codependence. Codependence was negatively correlated with socially desirable masculinity and femininity traits. Moreover, codependence was related to "Adult Children of Alcoholics" traits, shame, and vulnerability to depression [sociotropy]. Sensation seeking, negative masculinity, and problem drinking tendencies loaded on a separate factor called contradependence. These empirical findings suggest the utility of the codependence concept for further research in clinical or community settings, particularly when distinguished from contradependence.
\end{abstract}

Keywords Codependence, Contradependence, Problem Drinking, Gender-Stereotyped Traits, Sex Roles

\section{Introduction}

Codependency has been succinctly defined as a psychosocial condition characterized by "extreme focus outside of self, lack of open expression of feelings, and attempts to derive a sense of purpose through relationships" $[1$, p. 27]. This construct has been widely used in the mental health and chemical dependency fields [2]. While not universally accepted, codependency has nevertheless been carefully conceptualized and measured $[3,4,5]$. Recent studies continue to find the codependency concept useful, particularly when it is situated within a broader theoretical framework of personality theory and development $[6,7]$. Codependency has also been of recent interest in cross-cultural studies $[8,9,10]$.

Anderson [11] criticized the codependency concept on the grounds that it lacks diagnostic validity, emphasizes separation from family of origin rather than connection, pathologizes feminine qualities and behaviors, and neglects the unequal distribution of power and resources between males and females. Collins [4] also critiqued this concept as popularized in self-help movements and recommended that practitioners not use this model. Like Anderson, Collins claimed that codependency, used as an addictive disease model, blames the woman as "victim" and treats traditional femininity as pathological. Collins provides a feminist perspective which claims that women's emotional development occurs in a relational context: the "self-in-relation" theory would predict that women's "codependent" behaviors do not represent a failure to separate, but instead represent a conflict in "connecting" with significant others. The important implication is that codependent behaviors should be construed as positive, affiliative efforts.

Codependency is sometimes considered as a pathological label or stigmatizing status, typically assigned to women in relationships with men who engage in addictive behaviors. However, in this paper, we shall examine codependence as a dimension of personality, varying by degree from normality to deviance, as operationalized by gender-stereotyped attributes, which may be expressed by both women and men. This approach also emphasizes the coherence of this construct within a broader conceptual framework provided by psychological studies of attitudes and behaviors in both normal and clinical populations.

A comprehensive theoretical framework for codependence was developed by Hogg and Frank [3]. According to their model, emotional health results from interdependence, a balance along a continuum between the poles of affiliation and differentiation. In contrast, emotional neediness results when efforts for differentiation and affiliation are not met in healthy, socially desirable ways. Conceptualized along a separate and parallel continuum, codependence and contradependence are two poles representing unhealthy extremes corresponding to affiliation and differentiation, respectively. Hogg and Frank considered codependent behavior to be exhibited in socially undesirable stereotypically feminine gender roles, while 
contradependent behavior would be the counterpart for socially undesirable masculine gender-stereotyped roles. In other words, whereas codependence would involve a loss of boundaries and over-engagement with other people, contradependence is the tendency to disengage from others to avoid emotional pain. In this framework, codependence and contradependence are viewed as behavioral matrices for a variety of psychiatric diagnoses associated with these negative interpersonal strategies.

A problem with the Hogg and Frank model is that codependence and contradependence were not sufficiently operationalized such that measures were specified. For their theory to be useful in clinical research and practice, it is necessary to provide measurement tools for these broad concepts. Fortunately, the model of Hogg and Frank is strikingly reminiscent of Bakan's [12] philosophical formulation of "agency" and "communion." Spence and Helmreich [13] used Bakan's model as the metatheoretical basis for their measures of psychological masculinity [agency or instrumentality] and femininity [communion or expressiveness]. This model has been widely used in empirical studies of gender-stereotyped traits. As in the Hogg and Frank model, Spence, Helmreich, and Holahan [14] theorized both positive and negative manifestations of differentiation and affiliation. Their "positive masculinity" scale has items conveying socially desirable agency [or instrumentality], and achievement. The "positive femininity" items reflect socially desirable expressive stereotyped traits, using the Extended Personality Attribute Questionnaire [EPAQ] [15]. Spence and colleagues' "negative masculinity" [14] scale describes attributes that appear to be similar to contradependent patterns, while their "negative femininity" scales resemble codependent ones.

Cowan and Warren [5] empirically examined the relationship between codependency measures and positive and negative gender stereotyped traits, using the Extended Personality Attributes Questionnaire [EPAQ] [14]. They first factor analyzed items from codependency inventories developed by Beck [16] and by Potter-Efron and Potter-Efron [17]. The eight factors that resulted each had good internal consistency reliability and criterion validity in discriminating family members of chemically dependent patients in treatment from normative student controls. The codependency measures were then administered to 339 female and 115 male college students, $63 \%$ of whom were Caucasian. The students also completed five of the EPAQ scales: positive masculinity $[\mathrm{M}+]$, positive femininity $[\mathrm{F}+]$, negative masculinity [M-], and two negative femininity scales [FVA- and FC-], which measure verbal passive-aggressiveness and excessive communality.

Cowan and tested several hypotheses. First, they tested the prediction that women should obtain higher codependency scores than men. This hypothesis was not supported: women scored higher than men on only two of the eight codependency measures [Negative Affect/Low Self-Esteem, and Responsibility for Others], and there were no significant gender differences on the EPAQ negative femininity scales. Males scored higher on the negative masculinity scale. The second hypothesis was that codependency, as defined in their study, would be significantly associated with only the negative femininity scales on the EPAQ and that there would be no correlation with the positive femininity scales. This prediction was supported by the data, suggesting that "codependency does not overlap with valued female-stereotyped traits, but rather with negative feminine traits devalued in both women and men" [5, p. 631].

Dear and Roberts [7] have conceptually replicated the findings of Cowan and Warren [5]. Using a sample of Australian university students [ $\mathrm{N}=192]$, they administered the Holyoake Codependency Index [HCI] [18] and the Personal Description Questionnaire [PDQ] [19], which is conceptually equivalent to the EPAQ. The "external focus" [or approval seeking] dimension of codependency on the HCI was associated with high scores on socially undesirable, negative femininity and low scores on socially desirable, positive masculinity. The "self-sacrificing" dimension was related to high scores on socially desirable, positive femininity and low scores on socially undesirable, negative masculinity.

Although neither Cowan and Warren nor Dear and Roberts cite the Hogg and Frank paper, their findings would fit the Hogg and Frank interpersonal model of codependence-contradependence. Hogg and Frank theorized that socially valued differentiation - affiliation and socially devalued contradependence - codependence are parallel continua, the first associated with emotional health and the second with emotional neediness. A negative high correlation might be expected between opposite poles across the two continua [e.g., positive masculinity with codependence], while a low positive correlation might be expected between opposite poles along the same continuum [e.g., positive masculinity with positive femininity, or, negative masculinity with codependence].

One limitation of both the theoretical framework of Hogg and Frank and the empirical research [5,7] is that the discriminant validity of the codependency construct was not determined by operationalization of measures that would distinguish codependence from contradependence. Ruehling, Koelbel, and Rutgers [20] did distinguish codependence from conduct disorder in their study of feminine versus masculine coping responses to parental abuse. In their sample of 218 high school students, codependence was measured by their Codependent Questionnaire [CdQ] and the Fischer, Spann, and Crawford Codependent Questionnaire [21]. Codependence was associated with high scores on negative femininity and low scores on positive masculinity [using EPAQ definitions]. In contrast, conduct disorder [a continuous measure they derived from 22 DSM-IIIR behaviors] was related to high scores on negative masculinity. Conduct disorder would be conceptually similar to contradependence as defined in the Hogg and Frank model. 
The present study used Beck's Codependent Assessment Scale [16] and conceptually analogous standardized measures, i.e., the Adult Children of Alcoholics Tool [22] and the Internalized Shame Scale [23]. In addition, scales were selected that would differentially relate to feminine vs. masculine gender-stereotyped traits. In this fashion, the association between codependence and feminine negative traits, and the association between contradependence and masculine negative traits, as predicted by Hogg and Frank [3], could be explored.

To further elaborate the construct validity of the Hogg and Frank model, we investigated the possible relationship between codependence and contradependence with respect to depression and substance abuse susceptibility. Women are more frequently diagnosed with depression, while men are more at risk for depression $[24,25]$. The present study examines whether tendencies toward these clinical outcomes are more strongly related to differences in codependence contradependence rather than gender. Further, it explores whether children of alcoholics obtain higher scores on codependence and/or contradependence.

Robins and Luten [26] developed the Personality Style Inventory [PSI] as a measure of two essentially orthogonal vulnerability factors for depression: "sociotropy" and "autonomy." The sociotropy subscales ["concern with what others think," "dependency," and "pleasing others"] are suggestive of codependence, while the autonomy subscales ["perfectionism / self-criticism," "need for control," and "defensive separation"] appear similar to contradependence. Robins and Luten did not report the relationship between the PSI and measures of feminine and masculine personality attributes, such as the EPAQ scales. However, the sociotropy scale items appear to have face validity for feminine genderstereotypes, while the autonomy scale items [particularly the defensive separation items] suggest masculine gender-stereotypes.

The second area of potential relevance for exploring the convergent and discriminant validity of codependence and contradependence and their purported gender-stereotypes is substance abuse. The proportion of substance abusing [SA] males in the U.S. is about twice that for SA women, and only $21 \%$ of people treated for alcohol problems were female [27]. Some studies [28, 29], however, have suggested that gender roles [masculinity - femininity] may mediate the sex differences in drinking behavior. Several research studies emphasize the importance of the context of interpersonal relationships for understanding SA in women. Block, Block, and Keyes [30] found that early family environment was more closely related to drug abuse in girls than in boys. Harrison and Hoffman [31] showed that females with SA problems expressed lower self-esteem, more depression, and greater preoccupation with weight and appearance. In contrast, young males with SA problems reported higher self-regard, but more risk-taking and antisocial behavior. Cloninger [32] reported evidence from behavior and genetic studies that early SA onset in males is related to thrill or sensation seeking and antisocial tendencies. Zuckerman [33] developed a sensation-seeking scale to operationalize this construct, which appears to be conceptually similar to contradependence as described by Hogg and Frank.

There were two purposes for the present study. The first aim was to replicate and extend Cowan and Warren's study by examining the relationship between codependency, as defined by Beck's measure, and standardized measures of shame, depression, and adult children of alcoholics [ACOA] traits in a sample of undergraduates. We expected that students from problem drinking families [ACOAs] would score higher on codependency as well as on self-report measures of substance abuse. The second purpose was to elaborate the construct of codependency, using the interpersonal model of Hogg and Frank, by including not only feminine gender-stereotyped attributes [EPAQ $\mathrm{F}+$, FVA-, FC- scales, codependency, and sociotropy] but also masculine gender-stereotyped attributes [EPAQ $\mathrm{M}+, \mathrm{M}-$ scales, problem drinking tendencies, autonomy, and sensation seeking] in order to operationalize contradependence.

\section{Methodology}

Participants and Procedure: This convenience sample consisted of 208 undergraduate social work students [41 males, 167 females, 2 unspecified] who were enrolled in classes at a large southern public university in the USA. Their mean age was 23.6 years [ $\mathrm{SD}=5.6]$. The race/ethnicity of the sample was: 118 whites [56\%], 43 Hispanics [20.5\%], 19 African-Americans [9\%], and 11 other [4.7\%]. A student sample was chosen because the hypothesized correlates of codependence and contradependence were predicted to be analogous along the continuum between normality and deviance. This assumption permits the use of a student sample in preliminary correlational research.

The students were recruited by contacting faculty members teaching undergraduate social work classes and obtaining permission to administer the packet of questionnaires to volunteers during class time. This procedure was approved by the University IRB. The researcher met with each class to explain the nature of the study [i.e., "the relationship between adult functioning and your family of origin"], informed consent, and confidentiality safeguards. The professor and the researcher then left the room and students were instructed to leave their packets, completed or otherwise, in a designated place. At the end of class, the researcher returned to pick up their packets. This procedure produced a completion rate of $90 \%$ for the students surveyed.

Measures: The Codependence Assessment Scale (CODAS) [16] is a 39-item self-report scale developed by Beck. There are four cognitive-affective factors [Self-Expression, Independence, Identification/Social Concern, and Control] and three biographical factors [Family Acceptance, Family Tension, Family Chemical Dependence] that were identified though factor analysis. Higher scores on the CODAS factors 
indicate more codependence. Internal consistency reliability estimates [Cronbach's alpha coefficient] for the factors in the present sample were .7 or higher, with the exception of the Social Concern factor [.58]. For the CODAS total score the internal consistency reliability was .87 . Beck reported that self-described codependents differed significantly from student controls on these factors, providing some evidence for the criterion validity of this measure. Using the terminology of Cowan and Warren [5], the CODAS cognitive-affective factors would correspond to the Lack of Expression of Feelings scale, the Lack of Autonomy scale, the Responsibility for Others scale, and the Control of Others scale. Only one of the CODAS biographical factors [Family Acceptance], corresponds to a Cowan and Warren scale, Perceived Lack of Family Acceptance. The Cowan and Warren Dysfunctional Significant Other scale and the Parental Dysfunction scale included items from the CODAS Family Tension factor and Family Chemical Dependence factor. In the present study we will use the Cowan and Warren labels for the four CODAS cognitive-affective codependence scales and retain Beck's labels for the three CODAS biographical scales.

The Adult Children of Alcoholics Tool (ACAT) [22] consists of two parts. Part I of the ACAT [25-items] measures the personality characteristics of the Adult Children of Alcoholics [ACA] syndrome. None of the Part I items refers to a parent's drinking problem or alcoholism. The internal consistency reliability of the ACAT was .84 and the test-retest reliability was .78 in a student sample. Cronbach's alpha coefficient was .81 in the present study. The ACAT has been found to discriminatd ACAs from controls in both student and clinical samples [22], and the ACAT was significantly correlated [.62] with a conceptually similar measure, the Adult Children of Alcoholics Index [34]. Part II of the ACAT comprises five items that ask about the amount of alcohol consumed by each parent and the respondent, along with asking whether either parent[s] and/or grandparent[s] were regarded as having had a "drinking problem."

The Internalized Shame Scale (ISS) [23] is a 30-item questionnaire that measures the subjective experience of shame. The internal consistency reliability coefficient was reported to be .94 for an undergraduate sample [with a test-retest correlation of .84], and was .97 in the present study.

The Personal Style Inventory (PSI) [26] consists of 48 items developed to discern two vulnerability factors for depression: Sociotropy and Autonomy, each measured by 24 items. In this study, Version II of the PSI was used [35]. In an undergraduate sample the internal consistencies of the sociotropy and autonomy factors were .88 and .86 respectively [. 85 and .80 in the present study].

The Sensation Seeking Scale (SSS) [33] is a 40-item forced-choice measure of sensation seeking, developed in a student sample. In this study, Form $\mathrm{V}$ was used. The internal consistency reliability coefficient for the total scale calculated in the present study was .82 .
The Drinking Restraint Scale (DRS) [36] is a 7-item questionnaire used to identify students who believe they have to control or restrain their drinking tendencies for fear of becoming alcoholic. Reliability information was not originally reported; however, a later study [38] determined that the internal consistency was satisfactory [.73]. Cronbach's alpha was .71 in the present study.

The Self-Administered Short Michigan Alcoholism Screening Test (SMAST) [38] is a 13-item self-report screening test to detect possible alcoholism in the respondent. The reliability coefficient is reported to be .93 and was .71 in the present study.

The Extended Personal Attributes Scale (EPAQ) [15] is a 40-item self-report instrument for adults was developed using college students to measure personality attributes, found in both males and females, but stereotypically associated with a particular gender. Following Cowan and Warren [5], five EPAQ scales were used in the present study. Two scales [positive masculinity, $\mathrm{M}+$; positive femininity, $\mathrm{F}+]$ have items that measure gender-stereotyped traits that are socially desirable, and three scales [negative masculinity, M-; negative feminine verbal passive- aggressiveness, FVA-; negative feminine communion, FC-] include items that measure gender-stereotyped traits that are socially undesirable. The positive masculine $[\mathrm{M}+]$ scale contains attributes judged to be more characteristic of males than of females [e.g., independent, active, competitive] while the positive feminine $[\mathrm{F}+]$ scale has items considered to be more characteristic of females than of males [e.g., gentle, helpful, kind]. The $\mathrm{M}+$ scale is described [13] as a measure of instrumentality [agency] that is highly correlated with overall self-esteem. The $\mathrm{F}+$ scale is also related to self-esteem, although not so strongly as the $\mathrm{M}+$ scale, and it measures expressiveness toward others. As described by Cowan and Warren, the two socially undesirable feminine stereotypical trait scales, FC- and FVA-, appear to assess two aspects of codependency: subordinating oneself to others' needs [FC-][e.g., spineless, servile] and excessive control of others through complaining [FVA-][e.g., nagging, whiny]. The negative masculinity scale [M-], consisting of socially undesirable agency items [e.g., arrogant, egotistical, dictatorial], resembles the Hogg and Frank [3] contradependence construct: interpersonal control through independence [e.g., self-centeredness, feeling superior, greed, and bossiness]. The internal consistency reliabilities for the 8-item M+, F+, and M- scales and for the 4-item FCand FVA- scales have been reported to be $.74, .75, .69, .46$, and .60 , respectively [15]. The corresponding values in the present study were $.74, .76, .71, .48$, and .71 . The validity of the EPAQ scales has been well established in studies relating gender- stereotypes to measures of neurosis and acting out behavior [14], negative body image [39], and substance abuse $[28,29]$.

\section{Results}

Gender differences: A multivariate analysis of variance 
[MANOVA] was performed on the seven codependence [CODAS] scales with subject's gender as the independent variable. The MANOVA computation takes into account the number of dependent measures, grouping these when testing for statistical significance, thereby minimizing the risk of accumulated Type I error. The sample size in this study was sufficient to detect a medium effect size. The MANOVA main effect for gender on the CODAS subscales did not attain statistical significance $[\mathrm{F}[7,184]=1.48, \mathrm{p}=.178]$.

A second MANOVA of the effects of gender on the Spence-Helmreich EPAQ scales was significant $[F[5,183]=$ $9.95, \mathrm{p}<.001]$. Univariate ANOVAs revealed significant gender differences on the $\mathrm{M}+, \mathrm{F}+, \mathrm{M}-$, and FVA- scales. As shown in Table 1, females scored higher than males on the $\mathrm{F}+$ and FVA- scales, with a trend for higher scores on the FC- scale. Men scored significantly higher on the $\mathrm{M}+$ and $\mathrm{M}$ scales.

Table 1. Univariate analyses comparing means for females and males on the EPAQ gender-stereotyped trait scales ${ }^{\mathrm{a}}$.

\begin{tabular}{|c|c|c|c|c|c|}
\hline & \multicolumn{2}{|c|}{ Women } & \multicolumn{2}{c|}{ Men } & \\
\hline Scales & Mean & SD & Mean & SD & F Value \\
\hline M+ & 20.47 & 4.66 & 22.77 & 3.67 & $8.19^{* * *}$ \\
\hline F+ & 25.84 & 3.76 & 23.13 & 4.14 & $15.41 * * *$ \\
\hline M- & 11.11 & 4.23 & 13.05 & 5.18 & $5.93 *$ \\
\hline FVA- & 6.89 & 2.86 & 4.87 & 3.05 & $14.93^{* * *}$ \\
\hline FC- & 6.72 & 2.30 & 6.00 & 2.38 & 3.04 \\
\hline
\end{tabular}

a $\mathrm{N}$ females $=167, \mathrm{~N}$ males $=41, \mathrm{df}[1,187]$.

$* \mathrm{p}<.05$.

$* * \mathrm{p}<.01$.

$* * * \mathrm{p}<.001,1$-tailed].

Legend: $\mathrm{M}+=$ positive masculinity; $\mathrm{F}+=$ positive femininity; $\mathrm{M}-$ = negative masculinity; FVA- = negative feminine verbal-aggressiveness; FC- = negative feminine communality.

Table 2. Univariate analyses comparing means for females and males on several measures of personality and drinking behavior ${ }^{b}$.

\begin{tabular}{|c|c|c|c|c|c|}
\hline & \multicolumn{2}{|c|}{ Women } & \multicolumn{2}{c|}{ Men } & \\
\hline Scales & Mean & SD & Mean & SD & $\begin{array}{c}F \\
\text { Values }\end{array}$ \\
\hline ACAT & 23.97 & 11.90 & 21.50 & 7.46 & 1.10 \\
\hline ISS & 25.54 & 17.76 & 19.61 & 13.19 & 2.77 \\
\hline SOCIOTROPY & 95.56 & 15.21 & 91.61 & 13.65 & 1.59 \\
\hline AUTONOMY & 83.07 & 13.64 & 85.42 & 12.03 & .71 \\
\hline SSS & 17.12 & 5.94 & 20.64 & 6.66 & $7.68^{* *}$ \\
\hline DRS & 10.37 & 2.99 & 10.75 & 3.65 & .32 \\
\hline SMAST & .73 & 1.20 & 1.46 & 1.82 & $6.91 * *$ \\
\hline
\end{tabular}

${ }^{\mathrm{b}} \mathrm{N}$ females $=122, \mathrm{~N}$ males $=28$, df $[1,148]$.

$* \mathrm{p}<.05$.

$* * \mathrm{p}<.01$.

$* * * \mathrm{p}<.001$ [one-tailed].

Legend: ACAT $=$ Adult Children of Alcoholics Tool; ISS $=$ Internalized Shame Scale; SOCIOTROPY = Personality Style Inventory, Sociotropy Scale;

AUTONOMY = Personality Style Inventory, Autonomy Scale; SSS = Sensation Seeking Scale; DRS = Drinking Restraint Scale; SMAST = Short Michigan
Alcoholism Screening Test.

A third MANOVA was performed to examine gender effects on the other measures. This MANOVA was also significant $[\mathrm{F}[7,142]=3.10, \mathrm{p}=.005]$. The univariate ANOVAs [Table 2] indicate that significant gender differences occurred on only two measures, the Sensation Seeking Scale [SSS] and the Short Michigan Alcoholism Screening Test [SMAST]. In both cases men scored higher than women. There was a trend for women to score higher on the Internalized Shame Scale [ISS] [p=.098].

Some sample shrinkage occurred in the third MANOVA through the requirement for listwise deletion. T-tests for independent groups, with pairwise deletion of missing data, were performed, revealing an additional significant gender difference: women scored higher on the sociotropy factor of the PSI, $\mathrm{t}[187]=2.35, \mathrm{p}=.02$, 2-tailed test.

Family history of problem drinking: It was possible to determine family history of problem drinking $[\mathrm{FH}+]$ for 192 of the 208 students, based upon responses to Part II of the ACAT [22]. Fifty-two students [11 males, 40 females, 1 unspecified] were $\mathrm{FH}+$ for problem drinking. Additional MANOVAs were performed to explore whether the family history of problem drinking [FH+ vs. FH-], gender, and the interaction between gender and family history were related to the codependency scales, the EPAQ scales, and the other measures.

First, a multivariate analysis of variance [MANOVA] was performed on the seven codependence [CODAS] scales with subject's gender and family history [FH+ vs. $\mathrm{FH}-]$ as the independent variables. The MANOVA for the CODAS subscales for family history attained statistical significance $[F[7,182]=8.59, p=.000]$, but neither gender $[F[7,182]=$ $1.65, \mathrm{p}=.13]$ nor the interaction of gender and family history $[F[7,182]=.45, p=.87]$ were significant. Univariate $F$ tests revealed that four of the seven CODAS scales significantly differed by FH+ vs. FH- at the $\mathrm{p}<.01$ level. These scales were Responsibility for Others, Family Acceptance, Family Tension, and Family Chemical Dependency.

A second MANOVA revealed that family history of problem drinking did not significantly distinguish the Spence-Helmreich EPAQ scales $[\mathrm{F}[5,181]=1.09, \mathrm{p}=.37]$, nor did the interaction of gender and family history $[\mathrm{F}[5$, 181] $=.59, \mathrm{p}=.71]$. The gender effect was statistically significant, $[\mathrm{F}[5,181]=7.24, \mathrm{p}=.000]$, as described earlier.

A third MANOVA was performed to examine the effects of family history of problem drinking, gender, and their interaction, on the other measures. This MANOVA revealed a significant effect for family history $[F[7,140]=2.20$, $\mathrm{p}=.038]$. The univariate ANOVAs indicate that $\mathrm{FH}+$ students scored significantly higher on the Adult Children of Alcoholics Tool [ACAT], the Sensation Seeking Scale [SSS], the Drinking Restraint Scale [DRS], and tended to score higher on the PSI Autonomy scale [AUTONOMY] $[\mathrm{p}=.062$ ] and the Short Michigan Alcoholism Screening Test [SMAST] $[\mathrm{p}=.075]$. The gender effect was statistically significant $[F[7,140]=3.32, p=.003]$, as described earlier, but the interaction of gender and family history [FH+ vs. FH-] 
was not significant $[\mathrm{F}[7,140]=.79, \mathrm{p}=.60]$.

Correlations of CODAS and other measures with EPAQ gender-stereotyped traits:

Table 3 shows correlations between the CODAS codependence factors and the EPAQ gender-stereotyped trait scales. Because of the large number of intercorrelations, to reduce the probability of a Type I error, only those correlations in Table 3 with $\mathrm{p}<.01$ are treated as significant. Examination of Table 3 reveals that there were no significant positive relationships between the codependence factors and the socially desirable gender-stereotyped traits. Consistent with the findings of Cowan and Warren [5], four of the codependence scales as well as the total score [CODASTOT] were significantly negatively correlated with positive masculinity $[\mathrm{M}+]$. Lack of Self-expression, Lack of Family Acceptance, and CODASTOT were negatively correlated with positive femininity $[\mathrm{F}+]$. Contrary to expectation, only two of the codependence factors [Lack of Autonomy; Control of Others] were significantly associated with the socially undesirable gender- stereotyped traits [FVA-, FC-, and M-]. Cowan and Warren had reported that all eight of their codependency scales were significantly correlated with the FVA- and FC- scales; however, in the present study data the Beck CODAS scales were not consistently associated with negative femininity.

Table 4 and Table 5 extend the approach of Cowan and Warren [5] by exploring the convergent and discriminant validity of the codependence construct, in relationship to contradependence [as conceptualized by Hogg and Frank]. Beck's CODAS measure, the Adult Children of Alcoholics Tool [ACAT], the Internalized Shame Scale [ISS], and the Sociotropy factor of the Personality Style Inventory were all hypothesized to be significantly intercorrelated, as measures of codependence and of negative femininity. Table 4 shows that ACAT, ISS, and SOCIOTROPY were significantly positively correlated with the FC- scale and negatively correlated with $\mathrm{M}+$ [all p's <.01], and that these measures were significantly associated with the CODAS total score [Table 5].

Table 3. Correlations of CODAS Codependency and EPAQ gender-stereotyped trait scales ${ }^{\mathrm{a}, \mathrm{b}}$

\begin{tabular}{|c|c|c|c|c|c|}
\hline & \multicolumn{5}{|c|}{ Gender-Stereotyped Traits } \\
\hline Codependency Scales & $\mathrm{F}+$ & $\mathrm{M}+$ & FVA- & FC- & M- \\
\hline Lack of Expression & $-.33^{* *}$ & $-.29^{* *}$ & -.01 & .07 & .01 \\
\hline Lack of Autonomy & -.11 & $-.33^{* *}$ & -.07 & $.29^{* *}$ & -.04 \\
\hline $\begin{array}{c}\text { Responsibility } \\
\text { For Others } \\
\text { Control of Others }\end{array}$ & .09 & .12 & .13 & .00 & -.05 \\
\hline Family Acceptance & $-.18^{*}$ & -.08 & $.29^{* *}$ & $.28^{* *}$ \\
\hline Family Tension & -.12 & $-.20^{* *}$ & .06 & .04 & .14 \\
\hline Family CD & .06 & -.06 & -.04 & .07 & .14 \\
\hline CODAS Total Score & $-.17^{*}$ & $-.28^{* *}$ & .13 & .01 & .05 \\
\hline
\end{tabular}

a $\mathrm{N}=191$.

${ }^{\mathrm{b}}$ CODAS scales named by Cowan \& Martin labels [5], except for [Lack of] Family Acceptance, Family Tension, and Family Chemical Dependency.

${ }^{*} \mathrm{p}<.01, \quad 1$-tailed.

${ }^{* * *} \mathrm{p}<.001,1$-tailed.

Table 4. Correlations of other measures of personality and drinking behavior with the EPAQ gender-stereotyped trait scales a

\begin{tabular}{|c|c|c|c|c|c|}
\hline & \multicolumn{5}{|c|}{ Gender-Stereotyped Traits } \\
\hline Other Measure & $\mathrm{F}+$ & $\mathrm{M}+$ & $\mathrm{FVA}-$ & $\mathrm{FC}-$ & M- \\
\hline ACAT & -.11 & $-.35^{* *}$ & .04 & $.17^{*}$ & .06 \\
\hline ISS & -.04 & $-.44^{* *}$ & .08 & $.24^{* *}$ & -.02 \\
\hline SOCIOTROPY & $.29^{* *}$ & $-.34^{* *}$ & $.19^{*}$ & $.39^{* *}$ & -.15 \\
\hline AUTONOMY & $-.30^{* *}$ & -.12 & .06 & .01 & .15 \\
\hline SSS & -.15 & .04 & -.01 & $-.17^{*}$ & $.26^{* *}$ \\
\hline SMAST & .08 & .03 & .13 & -.02 & $.22^{* *}$ \\
\hline DRS & .02 & -.13 & .09 & .05 & .11 \\
\hline
\end{tabular}

a N's range from 151 to 191 .

${ }^{*} \mathrm{p}<.01$, 1-tailed.

${ }^{* *} \mathrm{p}<.001,1$-tailed. 
Table 5. Matrix of intercorrelations among the measures of personality and drinking behavior ${ }^{\mathrm{a}, \mathrm{b}}$

\begin{tabular}{|c|c|c|c|c|c|c|c|}
\hline Measures & ACAT & ISS & SOCIO & AUTON & SSS & SMAST & DRS \\
\hline CODASb & $.70^{* *}$ & $.49^{* *}$ & $.29^{* *}$ & $.28^{* *}$ & $.19^{*}$ & .18 & $.20^{*}$ \\
\hline ACAT & & $.55^{* *}$ & $.44^{* *}$ & $.50^{* *}$ & .18 & .16 & $.23^{*}$ \\
\hline ISS & & & $.40^{* *}$ & $.31^{* *}$ & .04 & .18 & $.28^{* *}$ \\
\hline PSISOCIO & & & & $.28^{* *}$ & -.01 & .09 & $.29^{* *}$ \\
\hline PSIAUTON & & & & & $.20^{*}$ & .09 & $.24^{*}$ \\
\hline SSS & & & & & & $.21^{*}$ & $.28^{* *}$ \\
\hline SMAST & & & & & & & $.64^{* *}$ \\
\hline
\end{tabular}

a N's range from 151 to 191 [pairwise deletion].

${ }^{\mathrm{b}}$ Total score on the Codependency Assessment Scales.

${ }^{*} \mathrm{p}<.01,1$-tailed.

${ }^{* *} \mathrm{p}<.001 .1$-tailed

Contradependence was operationalized by the EPAQ negative masculinity scale [M-], the Sensation Seeking scale, the PSI Autonomy scale, and the two measures of problem drinking. In Table 4 the Sensation Seeking scale [SSS] and the Short Michigan Alcoholism Screening Test [SMAST] were significantly positively associated with socially undesirable masculinity [M-] as predicted, while SSS was significantly negatively correlated with FC-. The correlations for the PSI Autonomy scale and for Drinking Restraint [DRS] with M- were positive, as predicted, but did not attain statistical significance at the .01 level. AUTONOMY was significantly negatively correlated with the $\mathrm{F}+$ scale and tended to be positively correlated with $\mathrm{M}$-, while SOCIOTROPY was significantly positively correlated with $\mathrm{F}+$ and tended to be negatively correlated with M-. This pattern suggests that the PSI Sociotropy and Autonomy factors may be particularly promising measures of codependence and contradependence, respectively. Inspection of Table 5 further reveals that AUTONOMY was significantly related to Sensation Seeking [SSS], but SSS was essentially uncorrelated with SOCIOTROPY.

Factor analysis: To simplify the examination of the relationships among the measures, a principal components factor analysis with varimax rotation was performed. Four factors, accounting for $61.6 \%$ of the total variance, were extracted [eigenvalues $>1.0$ ]. Table 6 shows the measures loading $>.3$ on each of the four factors. The first factor appears to involve general emotional dissatisfaction or low self-esteem. The CODAS, ACAT, ISS, and PSI scales all loaded positively on this factor while the $\mathrm{M}+$ scale loaded negatively. Sensation Seeking and the problem drinking measures loaded on the second factor, along with the Mscale. The third factor involves socially desirable femininity $[\mathrm{F}+]$ and sociotropy, with low negative masculinity and low sensation seeking. Interestingly, the Beck Codependence Assessment Scale total score does not load on this factor. The socially undesirable feminine gender stereotyped traits [FVA- and FC-] load on the fourth factor, along with Sociotropy and low negative masculinity.
Table 6. Principal components factor analysis [varimax rotation] on the EPAQ gender-stereotyped traits, CODAS total score, and other measures ${ }^{\text {a }}$

\begin{tabular}{|c|c|c|c|c|}
\hline Scale & Factor 1 & Factor 2 & Factor 3 & Factor 4 \\
\hline CODASTOT & .73 & & & \\
\hline ACAT & .87 & & & \\
\hline ISS & .74 & & & \\
\hline SOCIOTROPY & .46 & & .64 & .30 \\
\hline AUTONOMY & .60 & & & \\
\hline SSS & & .55 & -.31 & \\
\hline M+ & -.55 & & & -.37 \\
\hline F+ & & & .81 & \\
\hline FVA- & & & & .79 \\
\hline FC- & & & & .47 \\
\hline M- & & .34 & & \\
\hline SMAST & & .79 & & \\
\hline DRS & & .76 & & \\
\hline Eigenvalue & 3.34 & 1.88 & 1.45 & 1.33 \\
\hline \% Variance & 25.70 & 14.40 & 11.20 & 10.30 \\
\hline
\end{tabular}

${ }^{a}$ Only factor loadings $>.3$ are shown.

Regression analyses: Following Cowan and Warren [5], stepwise regressions were computed on the seven CODAS codependence scales using the five EPAQ gender-stereotyped traits and gender as the predictors. Table 7 shows the significant predictors, final beta weights, multiple correlations, and amount of variance accounted for by each scale. Gender-stereotyped traits were significant predictors for five of the seven CODAS codependence scales, but gender was never entered first in any of these regression equations, in agreement with Cowan and Warren [5]. One notable difference in these findings, in contrast to Cowan's and Warren's data, was that negative female gender-stereotyped traits [FVA- or FC-] entered significantly in only two of the seven regression equations for the codependency scales [i.e., for Lack of Autonomy and 
Control of Others]. Instead, negative masculinity [M-] was a significant predictor for three CODAS scales [i.e., Control of Others, Lack of Family Acceptance, Family Tension], while socially desirable gender-stereotyped traits $[\mathrm{F}+$ or $\mathrm{M}+]$ were significant negative predictors in four equations [i.e., Lack of Self-expression, Lack of Autonomy, Lack of Family Acceptance, and Family Tension].

Table 7. Stepwise regressions of CODAS Codependence Scales and other measures by Sex and EPAQ gender-stereotyped traits.

\begin{tabular}{|c|c|c|c|}
\hline \multicolumn{4}{|c|}{ Mult. R } \\
\hline & Beta & [Change] & R2 [Change] \\
\hline \multicolumn{4}{|c|}{ CODAS Lack of Expression } \\
\hline Step 1: F+ & -.30 & .33 & .11 \\
\hline Step 2: M+ & -.26 & .42 & .17 \\
\hline \multicolumn{4}{|c|}{ CODAS Lack of Autonomy } \\
\hline Step 1: M+ & -.32 & .33 & .11 \\
\hline Step 2: FC- & .23 & .38 & .14 \\
\hline Step 3: FVA- & -.21 & 43 & .18 \\
\hline \multicolumn{4}{|c|}{ CODAS Responsibility for Others } \\
\hline \multicolumn{4}{|c|}{ No Variables Entered Significantly } \\
\hline \multicolumn{4}{|c|}{ CODAS Control of Others } \\
\hline Step 1: FVA- & .17 & .30 & .09 \\
\hline Step 2: FC- & .22 & .37 & .13 \\
\hline Step 3: M- & .20 & .41 & .17 \\
\hline \multicolumn{4}{|c|}{ CODAS Lack of Family Acceptance } \\
\hline Step 1: M+ & -.25 & .23 & .05 \\
\hline Step 2: M- & .16 & .28 & .07 \\
\hline \multicolumn{4}{|c|}{ CODAS Family Tension } \\
\hline Step 1: M+ & -.18 & .20 & .04 \\
\hline Step 2: M- & .18 & .25 & .06 \\
\hline Step 3: Sex & .16 & .29 & .09 \\
\hline \multicolumn{4}{|c|}{ CODAS Family CD } \\
\hline \multicolumn{4}{|c|}{ No Variables Entered Significantly } \\
\hline \multicolumn{4}{|c|}{ CODAS Total Score } \\
\hline Step 1: M+ & -.29 & .28 & .08 \\
\hline Step 2: M- & .19 & .33 & .11 \\
\hline \multicolumn{4}{|c|}{ PSI Sociotropy } \\
\hline Step 1: FC- & .29 & .38 & .15 \\
\hline Step 2: F+ & .32 & .48 & .23 \\
\hline Step 3: M+ & -.29 & .55 & .30 \\
\hline \multicolumn{4}{|c|}{ PSI Autonomy } \\
\hline Step 1: F+ & -.30 & .30 & .09 \\
\hline \multicolumn{4}{|c|}{ Sensation Seeking Scale } \\
\hline Step 1: M- & .28 & .26 & .07 \\
\hline Step 2: FC- & .18 & .32 & .10 \\
\hline \multicolumn{4}{|c|}{ SMAST } \\
\hline Step 1: M- & .22 & .22 & .05 \\
\hline
\end{tabular}

Table 7 also summarizes the significant predictor variables for the total score on the Codependence Assessment Scales [CODASTOT], the PSI Autonomy and Sociotropy factors, the Sensation Seeking Scale, and the Short Michigan Alcoholism Screening Test. Most notable in these findings is that two of the measures predicted to operationalize the contradependence construct [i.e., SSS, SMAST] were significantly predicted by negative masculinity, while the PSI Sociotropy factor [hypothesized to be a measure of codependence vulnerability] was significantly predicted by negative feminine gender-stereotyped communion [FC-].

\section{Discussion}

Undoubtedly, chemical dependency counselors and self-help authors have overstated their case for codependency as a crucial construct for the treatment of family members of substance abusers. Nonetheless, based on the arguments and data presented in this paper, it may be premature to dismiss the codependence construct as a clinical practice model because of gender-stereotype bias, as recommended by Anderson [11] and Collins [4].

First, as shown by Cowan and Warren [5] and the present study, women who endorse traditionally feminine gender-stereotypes do not score differentially on measures of codependency. There were no gender differences discernible on the measures of codependency and adult children of alcoholics $[\mathrm{ACOA}]$ traits in the current study. In contrast, students reporting a family history of problem drinking $[\mathrm{FH}+]$ did score higher on codependence, as well as sensation seeking and concerns about drinking restraint. $\mathrm{FH}+$ students did not differ from FH- students on the EPAQ masculinity femininity scales. Males did score higher than females on sensation seeking and problem drinking, as measured by the Short Michigan Alcoholism Screening Test.

Second, gender-stereotyped personality traits [especially as operationalized by Spence and Helmreich, 13, 14, 15], found in both women and men, appear to tap aspects of the codependency construct, but only when codependence is reframed within a more complex theoretical framework as either the absence of socially desirable masculine agency or feminine expressive traits, or as the excess of socially undesirable feminine gender-stereotyped traits. Codependence, at least as operationalized in measures such as Beck's [16] Codependence Assessment Scales, appears to be factorially complex, possibly even including negative masculine stereotyped traits.

Third, while Anderson and Collins both advocate a self-empowerment model based on feminist theory and the self-in-relationship concept, this theoretical framework does not yet adequately address, nor operationalize, the dichotomy or polarity between agency and communion [or independence vs. dependence] in its socially desirable and undesirable aspects. Theorizing about codependence may require consideration of its conceptual opposite, 
contradependence, as modeled by Hogg and Frank [3]. Spence, Helmreich, and colleagues [13, 14, 15] have developed a measurement tool, the EPAQ, which appears to be compatible with the model of Hogg and Frank, including the provision of dual continua [i.e., socially desirable vs. socially undesirable forms of the same dimension].

Testing the adequacy of a more complex theory of codependence and contradependence [3] requires demonstration of construct validity [i.e., convergent and discriminant validity]. The present study provides preliminary data which indicate that codependence, operationalized as having more of negative feminine gender-stereotyped traits [particularly the FC- EPAQ scale], or as having less of socially desirable masculine and/or feminine stereotyped traits, was positively associated with higher scores on the PSI Sociotropy factor. Robins and Luten [26] hypothesized that Sociotropy was a vulnerability factor for interpersonally based depression. Preliminary discriminant validity was suggested by the finding that another construct, contradependence, could be operationalized by the Sensation Seeking scale and measures of drinking problem tendencies, and that sensation seeking/problem drinking would be associated with a different pattern of gender-stereotyped traits: negative masculinity. The results of the factor analysis and regression equations provide preliminary evidence in support of the construct validity of the distinction between codependence and contradependence.

The shortcomings of this study need to be clearly acknowledged. A sample of undergraduate social work students was drawn from a single university, limiting the generalizability of these findings, particularly for clinical or community samples. This convenience sample was predominantly female. It is possible that having a larger number of males may have resulted in finding significant gender differences for more of the dependent measures. However, Cowan and Warren [5] also used a student sample and found few gender differences in their study. Students were appropriate for this exploratory research because Hogg and Frank posited that the codependence - contradependence bipolar traits also varied along a second continuum of severity [from normal DSM "V" codes to personality disorders and addictions].

More studies using clinical or community samples are needed to replicate these preliminary data, especially the need to consider the cultural context of codependent vs. contradependent patterns. Collins and Anderson are correct to exhort practitioners and researchers to consider the importance of power differentials when proposing hypotheses about codependence. It may very well be the case that the codependence vs. contradependence distinction itself embodies the differences in power, as reflected in the masculine and feminine gender-stereotypes. Nevertheless, the preliminary findings from this study set the stage for further clinical research to determine the utility of the codependence and contradependence constructs for differential assessment and treatment applications.

Hogg and Frank illustrated some potential applications of their interpersonal model of codependence and contradependence that would be valuable for clinical research and practice. First, they argue that treating codependence and contradependence as behavioral matrices or dimensions, rather than discrete diagnoses, may assist counselors in forming "conceptual maps" [3, p. 374] which would distinguish among the "heterogeneity of causes, severities, and treatment modalities" [3, p. 374] being expressed as codependence or contradependence. Codependent and contradependent tendencies would be manifested along a continuum of severity as in the DSM-5 [25][i.e., from mild "V"-codes and adjustment disorders, to more serious diagnoses such as post-traumatic stress disorder, personality disorders, and addictions]. Differential etiologies would be implied for increasing severity of diagnosis. Hogg and Frank contend that use of their model would decrease the tendency to situate the problem in the codependent person, so as to not "blame the victim" [3, p. 374].

Second, Hogg and Frank contend that their model would facilitate specific treatment planning: "In much the same way that grief has many causes and levels of severity ... codependence and contradependence may assist counselors in the development of different treatment strategies" [3, p. 374]. For example, they describe how restoring a balance to relatedness would be therapeutic: "Clients with the contradependent pattern need to learn and experience safe and controlled ways of connecting ... [while those] with more codependent behavior need to experience and learn ways to care for themselves" [3, p. 374].

Third, Hogg and Frank point out that their model emphasizes the universality of the human needs to differentiate and connect. They contend that these emotional needs are manifested through stereotypically masculine and feminine gender roles that are healthy [i.e., differentiation, affiliation, or interdependence] or unhealthy [i.e., contradependence, codependence, or addictions]. Their model may facilitate developmental, social, and clinical studies of the value of socially desirable and socially undesirable gender stereotyped traits as protective or risk factors for predicting tendencies toward depression, substance abuse, and personality disorders. Such a comprehensive, theoretically derived and empirically supported framework would provide a strong foundation for the assessment and treatment of these conditions.

\section{Conclusions}

The present study provides further evidence for the convergent and discriminant validity of codependence conceptualized as a personality dimension which may have relevance for clinical research and practice, particularly when distinguished from contradependence. Codependence was negatively correlated with socially desirable masculinity 
and femininity traits. There were no gender differences on the codependence measures. Moreover, codependence was related to "Adult Children of Alcoholics" traits, shame, and vulnerability to depression [sociotropy]. Sensation seeking, negative masculinity, and problem drinking tendencies loaded on a separate factor we called contradependence.

When linked with appropriate measures, such as the ones used in this study, the Hogg and Frank interpersonal model of codependence and contradependence [3] may provide a framework for viewing the parallel continua of healthy and unhealthy agency and communion which may be useful for extending dimensional approaches to psychopathology and resilience.

\section{REFERENCES}

[1] L. Spann, \& J.L. Fischer. Identifying co-dependency. The Counselor, 8, 27, 1990.

[2] C. Hawkins, \& R.C. Hawkins II. Family systems and chemical dependency. In: C. A. McNeese \& D. DiNitto. [Eds.]. Chemical dependency: A systems approach. [4th edition] [pp.256-284]. Boston: Pearson, 2012

[3] J.A. Hogg, \& M.L. Frank. Toward an interpersonal model of codependence and contradependence. Journal of Counseling and Development, Vol.70, 371-375, 1992.

[4] B.G. Collins. Reconstruing codependency using self-in-relation theory: A feminist perspective. Social Work, Vol38, 470-476, 1993.

[5] G. Cowan, \& L.W. Warren. Codependency and gender-stereotyped traits. Sex Roles, Vol. 30, 631-645, 1994.

[6] L.L. Stafford. Is codependency a meaningful concept? Issues in Mental Health Nursing, Vol.22, 273-286, 2001.

[7] G.E. Dear, \& C.M. Roberts. The relationships between codependency and femininity and masculinity. Sex Roles, Vol.46, 159-165, 2002.

[8] S. Chang. A cultural perspective on codependency and its treatment. Asia Pacific Journal of Counseling and Psychotherapy, Vol. 3, 50-60, 2012.

[9] G.N. Gayol, \& L.R. Lira. Construction and validity of the codependency instrument [ICOD] for Mexican women. Salud Mental, Vol. 25, 38-48, 2002.

[10] S. Kwon. Codependence and interdependence: Cross-cultural reappraisal of boundaries and relationality. Pastoral Psychology, Vol. 50, 39-52, 2001.

[11] S.C. Anderson. A critical analysis of the concept of codependency. Social Work, Vol. 39, 677-685, 1994.

[12] D. Bakan. The duality of human existence. Boston: Beacon Press, 1966.

[13] J.T. Spence, \& R.L. Helmreich. Masculinity and femininity: Their psychological dimensions, correlates, and antecedents. Austin, TX: University of Texas Press, 1978.

[14] J.T. Spence, R.L. Helmreich, \& C.K. Holahan. Negative and positive components of psychological masculinity and femininity and their relationship to self-reports of neurotic and acting out behavior. Journal of Personality and Social Psychology, Vol. 37, 1673-1682, 1979.

[15] R.L. Helmreich, J.T. Spence, J. T., \& J.A.Wilhelm. A psychometric analysis of the Personal Attributes Questionnaire. Sex Roles, Vol. 7, 1097-1109, 1981.

[16] W.H. Beck. William Beck Assessment Scale Manual. Chicago, IL: Administrative Services, 1991.

[17] R.T. Potter-Efron, \& P.S. Potter-Efron. Outpatient co-dependency treatment. Special issue: Co-dependency: Issues in treatment and recovery. Alcoholism Treatment Quarterly, Vol. 6, 151-167, 1989.

[18] G.E. Dear, \& C.M. Roberts. The Holyoake Codependency Index: Investigation of the factor structure and psychometric properties. Psychological Reports, Vol. 87, 991-1002, 2000.

[19] J.K. Antill, J.D. Cunningham, G. Russell, \& N.L.Thompson. An Australian sex- role scale. Australian Journal of Psychology, Vol. 33, 169-183, 1981.

[20] P.V. Ruehling, N. Koelbel, \& C. Rutgers. Codependence and conduct disorder: Feminine and masculine coping responses to abusive parenting practices. Sex Roles, Vol. 35, 603-618, 1996.

[21] J.L. Fischer, L. Spann, \& D. Crawford. Measuring codependency. Alcoholism Treatment Quarterly, Vol. 8, 87-99, 1991.

[22] C.A. Hawkins, \& R.C. Hawkins II. Development and validation of an Adult Children of Alcoholics Tool. Research on Social Work Practice, Vol. 5, 317-339, 1995.

[23] D. Cook. Manual for the Internalized Shame Scale. Stout, WI: University of Wisconsin-Stout, 1990

[24] American Psychiatric Association. Diagnostic and statistical manual of mental disorders, Fourth Edition, Text Revision. Washington, DC: APA, 2000.

[25] American Psychiatric Association. Diagnostic and statistical manual of mental disorders, Fifth Edition. Washington, DC: APA, 2013.

[26] C.J. Robins, \& A.G. Luten. Sociotropy and autonomy: Differential patterns of clinical presentation in unipolar depression. Journal of Abnormal Psychology, Vol. 100, 74-77, 1991.

[27] S. Blume. Why are we concerned?: An overview of women and alcohol. The Counselor, Vol. 7, 14-20, 1989.

[28] W.E. Snell, S.S. Belk, \& R.C. Hawkins II. Alcohol and drug use in stressful times: The influence of the masculine role and sex-related personality attributes. Sex Roles, Vol. 16, 359-373, 1987.

[29] R.F. Huselid, \& M.L. Cooper. Gender roles as mediators of sex differences in adolescent alcohol use and abuse. Journal of Health and Social Behavior, Vol. 33, 348-362, 1992.

[30] J. Block, J. Block, \& S. Keyes. Longitudinally foretelling drug usage in adolescence: Early childhood personality and environmental precursors. Child Development, Vol. 59, 336-355, 1988. 
[31] P. Harrison, \& N. Hoffman, N. Chemical dependency in today's adolescents: A report on the 1989 Minnesota Student Survey. The Counselor, Vol. 8, 22-23, 1990.

[32] C.B. Cloninger. Neurogenetic adaptive mechanisms in alcoholism. Science, Vol. 236, 410-416, 1987.

[33] M. Zuckerman. Sensation seeking: Beyond the optimal level of arousal. Hillsdale, NJ: Lawrence Earlbaum Press, 1979.

[34] E.W. Gondolf, \& R.J. Ackerman, R. J. Validity and reliability of an "Adult Children of Alcoholics" Index. International Journal of the Addictions, Vol. 28, 257-269, 1993.

[35] C.G. Robins. Personal Style Inventory, Version II. Durham, NC: Duke University Medical Center, 1991.
[36] L. Bensley. The heightened role of external responsiveness in alcohol consumption of restrained drinkers. Cognitive Therapy and Research, Vol. 13, 623-636, 1989.

[37] C.A. Hawkins. Pathogenic and protective relations in alcoholic families [II]: Ritual invasion, shame, ACOA traits, and problem drinking in adult offspring. Journal of Family Social Work, Vol. 1, 51-63, 1996.

[38] M. Seltzer, A.Vinokur, \& L. van Rooijen. A self-administered Short Michigan Alcoholism Screening Test. Journal of Studies on Alcohol, Vol. 36, 117-126, 1975.

[39] R.C. Hawkins II, S. Turell, \& L.J. Jackson. Desirable and undesirable masculine and feminine traits in relation to students' dieting tendencies and body image dissatisfaction. Sex Roles, Vol. 9, 705-718, 1983. 